\title{
A SURVEY ON DIFFERENT TYPES OF GENERALIZED RECURRENCY ON THE WEYL MANIFOLD
}

\author{
Assist.Prof.Dr.Füsun NURCAN BAŞTAN \\ Marmara University, \\ The Department of Mathematics, \\ Faculty of Science and Letters, TURKEY. \\ funal@marmara.edu.tr
}

February 15, 2014

\begin{abstract}
Hayden [1] introduced a semi symmetric metric connection on the Riemannian manifolds. This definition was developed by Yano [2] and Imai [3-4]. In [5], we examined the Weyl manifold, which is the generalization of the Riemannian manifold, admitting a semi symmetric connection. Pavel Enghish [6-7] examined the recurrency and generalized recurrency on the Riemannian manifolds with respect to symmetric and semi symmetric connections.

The aim of this paper is to obtain the relations between different types of the Weyl manifolds under some special conditions which are concerning the definitions of generalized recurrency, generalized conformally recurrency and generalized projectively recurrency.
\end{abstract}

Keywords: Weyl manifold, semi symmetric connection, conformal curvature tensor, projective curvature tensor, generalized recurrency.

\section{Mathematical Subject Classification: 53A40}

\section{Introduction}

An n-dimensional manifold which has a symmetric connection $\nabla$ and a conformal metric tensor $g_{i j}$ is said to be Weyl manifold, if the compatible condition is in the form of

$$
\nabla_{k} g_{i j}-2 g_{i j} T_{k}=0
$$

where $T_{k}$ is a covariant vector field. Such a Weyl manifold will be denoted by $W_{n}\left(g_{i j}, T_{k}\right)[8]$. 
The coefficients $\Gamma_{j k}^{i}$ and $\bar{\Gamma}_{j k}^{i}$ of the symmetric connection $\nabla$ and the semi symmetric connection $\bar{\nabla}$, respectively, on the Weyl manifold are related by $[5]$

$$
\bar{\Gamma}_{j k}^{i}=\Gamma_{j k}^{i}+\delta_{k}^{i} S_{j}-g_{j k} S^{i}
$$

where $S_{i}=-2 a_{i}$ and $a_{i}$ is an arbitrary covariant vector. by

The torsion tensor $T_{j k}^{i}$ with respect to semi symmetric connection is defined

$$
T_{j k}^{i}=\delta_{k}^{i} S_{j}-\delta_{j}^{i} S_{k}
$$

The relation between the mixed curvature tensors $R_{i j k}^{h}$ and $\bar{R}_{i j k}^{h}$ of $\nabla$ and $\bar{\nabla}$ is obtained as

$$
\bar{R}_{i j k}^{h}=R_{i j k}^{h}+\delta_{k}^{h} S_{i j}-\delta_{j}^{h} S_{i k}+g_{i j} g^{h l} S_{l k}-g_{i k} g^{h l} S_{l j}
$$

where

$$
S_{i j}=\nabla_{j} S_{i}-S_{i} S_{j}+\frac{1}{2} g_{i j} g^{k l} S_{k} S_{l}
$$

and $\nabla_{j} S_{i}$ denotes the covariant derivative of $S_{i}$ with respect to $\nabla$.

By transvecting the equation (1.3) by $g_{m h}$ and contracting on the indices $h$ and $k$ in (1.3), we have the following relations, respectively,

$$
\begin{aligned}
& \bar{R}_{m i j k}=R_{m i j k}+g_{m k} S_{i j}-g_{m j} S_{i k}+g_{i j} S_{m k}-g_{i k} S_{m j}, \\
& \bar{R}_{i j}=R_{i j}+(n-2) S_{i j}+S g_{i j}, \text { where } S=g^{m k} S_{m k}
\end{aligned}
$$

where $R_{i j}$ and $\bar{R}_{i j}$ denote the Ricci tensors of $\nabla$ and $\bar{\nabla}$.

By using the definitions of $R$ and $\bar{R}$, we get

$$
\bar{R}=R+2(n-1) S
$$

where $R$ and $\bar{R}$ denote the scalar curvatures of $\nabla$ and $\bar{\nabla}$, respectively.

\section{The Generalized Recurrent Weyl Manifolds}

In this paper, the concepts of $W$ manifold and WS manifold mean Weyl manifolds admitting the symmetric connection $\nabla$ and the semi symmetric connection $\bar{\nabla}$, respectively.

Definition 1 The Weyl manifold is called recurrent WS manifold, if the mixed curvature tensor $\bar{R}_{i j k}^{h}$ of $\bar{\nabla}$ satisfies the condition

$$
\bar{\nabla}_{r} \bar{R}_{i j k}^{h}=\bar{\lambda}_{r} \bar{R}_{i j k}^{h}
$$

where $\bar{\lambda}_{r}$ is the recurrency vector of WS manifold. 
If it satisfies the condition

$$
\bar{\nabla}_{r} \bar{R}_{i j k}^{h}=\bar{\varphi}_{r} \bar{R}_{i j k}^{h}+\bar{a}_{r} \bar{K}_{i j k}^{h}
$$

where $\bar{K}_{i j k}^{h}$ is the generalized recurrency tensor, then the Weyl manifold is said to be generalized recurrent WS manifold. In (2.2), $\bar{\varphi}_{r}$ and $\bar{a}_{r}$ are the generalized recurrency vectors of WS manifold.

According to the Definition 1, it can be easily seen that each recurrent WS manifold is generalized recurrent. However, the converse of the given statement is not true in general case. So we prove a necessary and sufficient condition for a generalized recurrent WS manifold to be recurrent by the following theorem:

Theorem 2 A generalized recurrent WS manifold is recurrent if and only if the generalized recurrency tensor $\bar{K}_{i j k}^{h}$ is proportional to the curvature tensor $\bar{R}_{i j k}^{h}$ of $\bar{\nabla}$.

Proof. Suppose that a generalized recurrent WS manifold is recurrent. Then, equaling (2.1) and (2.2) leads to

$$
\bar{K}_{i j k}^{h}=\left[\frac{(\bar{\lambda}-\bar{\varphi})}{\bar{a}}\right] \bar{R}_{i j k}^{h}
$$

where $\bar{a}_{r} \bar{a}^{r}=\bar{a}$ and $\left(\bar{\lambda}_{r}-\bar{\varphi}_{r}\right) \bar{a}^{r}=\bar{\lambda}-\bar{\varphi}$.

Conversely; for a generalized recurrent WS manifold, suppose that the generalized recurrency tensor is proportional to the curvature tensor $\bar{R}_{i j k}^{h}$ of $\bar{\nabla}$. Then, we have (2.2) and

$$
\bar{K}_{i j k}^{h}=\alpha \bar{R}_{i j k}^{h}
$$

From (2.2) and (2.4), we obtain

$$
\bar{\nabla}_{r} \bar{R}_{i j k}^{h}=\bar{\lambda}_{r} \bar{R}_{i j k}^{h} \quad\left(\bar{\lambda}_{r}=\bar{\varphi}_{r}+\alpha \bar{a}_{r}\right)
$$

which completes the proof.

Definition 3 The Weyl manifold satisfying the condition

$$
\bar{\nabla}_{r} \bar{R}_{i j}=\bar{\varphi}_{r} \bar{R}_{i j}+\bar{a}_{r} \bar{K}_{i j}
$$

is called generalized Ricci recurrent WS manifold.

The Weyl manifold is called generalized scalar recurrent WS manifold, if it satisfies the condition

$$
\bar{\nabla}_{r} \bar{R}=\bar{\varphi}_{r} \bar{R}+\bar{a}_{r} \bar{K}
$$

Lemma 4 A generalized recurrent WS manifold is generalized Ricci recurrent and a generalized Ricci recurrent WS manifold is also generalized scalar recurrent. 
Definition 5 The Weyl manifold is called generalized recurrent $W$ manifold, if the mixed curvature tensor $R_{i j k}^{h}$ of $\nabla$ satisfies the condition

$$
\nabla_{r} R_{i j k}^{h}=\varphi_{r} R_{i j k}^{h}+a_{r} K_{i j k}^{h}
$$

where $K_{i j k}^{h}$ is the generalized recurrency tensor of $W$ manifold. In (2.7), $\varphi_{r}$ and $a_{r}$ are the generalized recurrency vectors of $W$ manifold.

Theorem 6 A recurrent WS manifold with the tensor $S_{i j}$ satisfying $\bar{\nabla}_{r} S_{i j}=$ $\bar{\lambda}_{r} S_{i j}$, where $\bar{\lambda}_{r}$ is the recurrency vector of WS manifold, is a generalized recurrent $W$ manifold.

Proof. By differentiating the curvature tensor $\bar{R}_{i j k}^{h}$ covariantly with respect to $\bar{\nabla}$, we obtain

$$
\bar{\nabla}_{r} \bar{R}_{i j k}^{h}=\bar{\nabla}_{r} R_{i j k}^{h}+\delta_{k}^{h}\left(\bar{\nabla}_{r} S_{i j}\right)-\delta_{j}^{h}\left(\bar{\nabla}_{r} S_{i k}\right)+g_{i j} g^{h l}\left(\bar{\nabla}_{r} S_{l k}\right)-g_{i k} g^{h l}\left(\bar{\nabla}_{r} S_{l j}\right)
$$

where $\bar{\nabla}_{r} R_{i j k}^{h}$ denotes the covariant derivative of the curvature tensor $R_{i j k}^{h}$ with respect to $\bar{\nabla}$.

Substituting the covariant derivative of the curvature tensor $R_{i j k}^{h}$ with respect to $\bar{\nabla}$ given by

$$
\bar{\nabla}_{r} R_{i j k}^{h}=\nabla_{r} R_{i j k}^{h}+R_{i j k r}^{h}
$$

where

$$
\begin{aligned}
R_{i j k r}^{h} & =S^{m}\left(R_{m j k}^{h} g_{i r}+R_{i m k}^{h} g_{j r}+R_{i j m}^{h} g_{k r}\right)-\left(R_{r j k}^{h} S_{i}+R_{i r k}^{h} S_{j}+R_{i j r}^{h} S_{k}\right) \\
& +R_{i j k}^{m}\left(\delta_{r}^{h} S_{m}-g_{m r} S^{h}\right)
\end{aligned}
$$

into (2.8), we get

$$
\begin{aligned}
& \bar{\nabla}_{r} \bar{R}_{i j k}^{h}-\delta_{k}^{h}\left(\bar{\nabla}_{r} S_{i j}\right)+\delta_{j}^{h}\left(\bar{\nabla}_{r} S_{i k}\right)-g_{i j} g^{h l}\left(\bar{\nabla}_{r} S_{l k}\right)+g_{i k} g^{h l}\left(\bar{\nabla}_{r} S_{l j}\right) \\
& =\nabla_{r} R_{i j k}^{h}+R_{i j k r}^{h} .
\end{aligned}
$$

By using $\bar{\nabla}_{r} S_{i j}=\bar{\lambda}_{r} S_{i j}$ and $(2.1),(2.10)$ reduces to

$$
\nabla_{r} R_{i j k}^{h}=\bar{\lambda}_{r} R_{i j k}^{h}-R_{i j k r}^{h}
$$

which is compatible with the Definition 5.

\section{The Generalized Conformally Recurrent Weyl Manifolds}

The conformal curvature tensor $\bar{C}_{i j k}^{h}$ of $\bar{\nabla}$ on the Weyl manifold is defined by $[5]$

$$
\bar{C}_{i j k}^{h}=\bar{R}_{i j k}^{h}-\frac{1}{n} \delta_{i}^{h} \bar{R}_{l j k}^{l}+\frac{1}{n-2} \bar{A}_{i j k}^{h}-\frac{1}{n(n-2)} \bar{B}_{i j k}^{h}-\frac{\bar{R}}{(n-1)(n-2)} G_{i j k}^{h}
$$


where

$$
\begin{gathered}
\bar{A}_{i j k}^{h}=\delta_{j}^{h} \bar{R}_{i k}-\delta_{k}^{h} \bar{R}_{i j}-g_{i j} g^{m h} \bar{R}_{m k}+g_{i k} g^{m h} \bar{R}_{m j}, \\
\bar{B}_{i j k}^{h}=\delta_{j}^{h} \bar{R}_{l k i}^{l}-\delta_{k}^{h} \bar{R}_{l j i}^{l}-g_{i j} g^{m h} \bar{R}_{l k m}^{l}+g_{i k} g^{m h} \bar{R}_{l j m}^{l}, \\
G_{i j k}^{h}=\delta_{j}^{h} g_{i k}-\delta_{k}^{h} g_{i j} .
\end{gathered}
$$

The conformal curvature tensors $C_{i j k}^{h}$ and $\bar{C}_{i j k}^{h}$ of the connections $\nabla$ and $\bar{\nabla}$ are related by [5]

$$
\bar{C}_{i j k}^{h}=C_{i j k}^{h}
$$

Definition 7 The Weyl manifold is called generalized conformally recurrent WS manifold, if the conformal curvature tensor $\bar{C}_{i j k}^{h}$ of the connection $\bar{\nabla}$ satisfies the condition

$$
\bar{\nabla}_{r} \bar{C}_{i j k}^{h}=\bar{\chi}_{r} \bar{C}_{i j k}^{h}+\bar{c}_{r} \bar{M}_{i j k}^{h}
$$

where $\bar{\chi}_{r}$ and $\bar{c}_{r}$ are the generalized conformally recurrency vectors of WS manifold having $\bar{M}_{i j k}^{h}$ as the generalized conformally recurrency tensor.

Lemma 8 A generalized recurrent WS manifold is generalized conformally recurrent.

Theorem 9 A generalized conformally recurrent WS manifold is generalized recurrent with the same recurrency vectors if and only if WS manifold is generalized Ricci recurrent and $\bar{M}_{i j k}^{h}$ is defined by

$$
\begin{aligned}
\bar{M}_{i j k}^{h} & =\bar{K}_{i j k}^{h}-\frac{1}{n} \delta_{i}^{h} \bar{K}_{l j k}^{l}+\frac{1}{n-2}\left\{\delta_{j}^{h} \bar{K}_{i k}-\delta_{k}^{h} \bar{K}_{i j}-g_{i j} g^{m h} \bar{K}_{m k}+g_{i k} g^{m h} \bar{K}_{m j}\right\} \\
& -\frac{1}{n(n-2)}\left\{\delta_{j}^{h} \bar{K}_{l k i}^{l}-\delta_{k}^{h} \bar{K}_{l j i}^{l}-g_{i j} g^{m h} \bar{K}_{l k m}^{l}+g_{i k} g^{m h} \bar{K}_{l j m}^{l}\right\} \\
& -\frac{\bar{K}}{(n-1)(n-2)}\left(\delta_{j}^{h} g_{i k}-\delta_{k}^{h} g_{i j}\right)
\end{aligned}
$$

where $\bar{K}_{i j k}^{h}$ is the generalized recurrency tensor of WS manifold.

Proof. Suppose that a generalized conformally recurrent WS manifold is generalized recurrent with the same recurrency vectors $\bar{\chi}_{r}$ and $\bar{c}_{r}$. Then, by Lemma 4, WS manifold is also generalized Ricci recurrent. By substituting (3.1), (2.2) and (2.5) in Definition 7, $M_{i j k}^{h}$ is obtained as given in the theorem.

Conversely, let a generalized conformally recurrent WS manifold be generalized Ricci recurrent with the same recurrency vectors $\bar{\chi}_{r}, \bar{c}_{r}$ and $\bar{M}_{i j k}^{h}$ be as in the theorem. In this case, we have (3.3) and from (2.5)

$$
\bar{\nabla}_{r} \bar{R}_{i j}=\bar{\chi}_{r} \bar{R}_{i j}+\bar{c}_{r} \bar{K}_{i j}
$$


By substituting (3.1), (3.5) and (3.4) in Definition 7, we get

$$
\bar{\nabla}_{r} \bar{R}_{i j k}^{h}=\bar{\chi}_{r} \bar{R}_{i j k}^{h}+\bar{c}_{r} \bar{K}_{i j k}^{h}
$$

which completes the proof.

Definition 10 The Weyl manifold is called conformally recurrent $W$ manifold, if the conformal curvature tensor $C_{i j k}^{h}$ of $\nabla$ satisfies the condition

$$
\nabla_{r} C_{i j k}^{h}=\beta_{r} C_{i j k}^{h}
$$

where $\beta_{r}$ is the conformally recurrency vector of $W$ manifold.

Similarly, if the conformal curvature tensor $\bar{C}_{i j k}^{h}$ of $\bar{\nabla}$ satisfies the condition

$$
\bar{\nabla}_{r} \bar{C}_{i j k}^{h}=\bar{\beta}_{r} \bar{C}_{i j k}^{h}
$$

where $\bar{\beta}_{r}$ is the conformally recurrency vector, the Weyl manifold is called conformally recurrent WS manifold.

Definition 11 The Weyl manifold is called generalized conformally recurrent $W$ manifold, if the conformal curvature tensor $C_{i j k}^{h}$ of $\nabla$ satisfies the condition

$$
\nabla_{r} C_{i j k}^{h}=\chi_{r} C_{i j k}^{h}+c_{r} M_{i j k}^{h}
$$

where $M_{i j k}^{h}$ is the generalized conformally recurrency tensor of $W$ manifold. In (3.8), $\chi_{r}$ and $c_{r}$ are the generalized conformally recurrency vectors of $W$ manifold.

Theorem 12 Each conformally recurrent WS manifold is a generalized conformally recurrent $W$ manifold. Similarly, each conformally recurrent $W$ manifold is a generalized conformally recurrent WS manifold.

Proof. Suppose that WS manifold is conformally recurrent. By (3.2), we get

$$
\bar{\nabla}_{r} \bar{C}_{i j k}^{h}=\bar{\nabla}_{r} C_{i j k}^{h}
$$

By using the covariant derivative of the conformal curvature tensor $C_{i j k}^{h}$ with respect to $\bar{\nabla}$, we have

$$
\bar{\nabla}_{r} C_{i j k}^{h}=\nabla_{r} C_{i j k}^{h}+C_{i j k r}^{h}
$$

where

$$
\begin{aligned}
C_{i j k r}^{h} & =S^{m}\left(C_{m j k}^{h} g_{i r}+C_{i m k}^{h} g_{j r}+C_{i j m}^{h} g_{k r}\right)-\left(C_{r j k}^{h} S_{i}+C_{i r k}^{h} S_{j}+C_{i j r}^{h} S_{k}\right) \\
& +C_{i j k}^{m}\left(\delta_{r}^{h} S_{m}-g_{m r} S^{h}\right) .
\end{aligned}
$$


By substituting (3.10) into (3.9), it is obtained as

$$
\bar{\nabla}_{r} \bar{C}_{i j k}^{h}=\nabla_{r} C_{i j k}^{h}+C_{i j k r}^{h}
$$

By using (3.7) and (3.2) in (3.11), we obtain

$$
\nabla_{r} C_{i j k}^{h}=\bar{\beta}_{r} C_{i j k}^{h}-C_{i j k r}^{h} .
$$

According to Definition 11, the above equation means that we obtain a generalized conformally recurrent $\mathrm{W}$ manifold.

For proving the second part of the theorem, suppose that $\mathrm{W}$ manifold is conformally recurrent. By using (3.6) and (3.2) in (3.11), we get

$$
\bar{\nabla}_{r} \bar{C}_{i j k}^{h}=\beta_{r} \bar{C}_{i j k}^{h}+C_{i j k r}^{h}
$$

showing that conformally recurrent $\mathrm{W}$ manifold is a generalized conformally recurrent WS manifold.

\section{The Generalized Projectively Recurrent Weyl Manifolds}

The projective curvature tensor $\bar{W}_{i j k}^{h}$ of $\bar{\nabla}$ on the Weyl manifold is defined as follows [5]:

$\bar{W}_{i j k}^{h}=\bar{R}_{i j k}^{h}+\frac{\delta_{i}^{h}}{n+1}\left\{\left(\bar{R}_{j k}-\bar{R}_{k j}\right)+2(n-1) \nabla_{[j} S_{k]}\right\}+\frac{1}{n^{2}-1}\left(\delta_{j}^{h} \bar{H}_{i k}-\delta_{k}^{h} \bar{H}_{i j}\right)$

where

$$
\bar{H}_{i j}=n \bar{R}_{i j}+\bar{R}_{j i}+2(n-1) \nabla_{[j} S_{i]} .
$$

By using the equations (1.3) and (1.6), we get the following relation between projective curvature tensors $W_{i j k}^{h}$ and $\bar{W}_{i j k}^{h}$ of the symmetric and semi symmetric connections $\nabla$ and $\bar{\nabla}$ by

$\bar{W}_{i j k}^{h}=W_{i j k}^{h}+\frac{2}{n+1} \delta_{i}^{h} \nabla_{[j} S_{k]}+\frac{1}{n^{2}-1}\left(\delta_{k}^{h} K_{i j}-\delta_{j}^{h} K_{i k}\right)+g_{i j} g^{h l} S_{l k}-g_{i k} g^{h l} S_{l j}$

where

$$
K_{i j}=n S_{i j}+S_{j i}+(n+1) S g_{i j} .
$$

Definition 13 The Weyl manifold is called generalized projectively recurrent WS manifold, if the projective curvature tensor $\bar{W}_{i j k}^{h}$ of the connection $\bar{\nabla}$ satisfies the condition 


$$
\bar{\nabla}_{r} \bar{W}_{i j k}^{h}=\bar{\phi}_{r} \bar{W}_{i j k}^{h}+\bar{d}_{r} \bar{N}_{i j k}^{h}
$$

where $\bar{\phi}_{r}$ and $\bar{d}_{r}$ are the generalized projectively recurrency vectors of WS manifold having $\bar{N}_{i j k}^{h}$ as the generalized projectively recurrency tensor.

Theorem 14 If the vector $S_{k}$ is gradient in a generalized recurrent WS manifold, then the manifold is generalized projectively recurrent.

Proof. If the vector $S_{k}$ is gradient in the WS manifold, then by differentiating the projective curvature tensor $\bar{W}_{i j k}^{h}$ given by (4.1) covariantly with respect to $\bar{\nabla}$ and applying the condition of generalized recurrency, we find

$$
\begin{aligned}
\bar{\nabla}_{r} \bar{W}_{i j k}^{h} & =\left(\bar{\varphi}_{r} \bar{R}_{i j k}^{h}+\bar{a}_{r} \bar{K}_{i j k}^{h}\right) \\
& +\frac{\delta_{i}^{h}}{n+1}\left\{\left(\bar{\varphi}_{r} \bar{R}_{j k}+\bar{a}_{r} \bar{K}_{j k}\right)-\left(\bar{\varphi}_{r} \bar{R}_{k j}+\bar{a}_{r} \bar{K}_{k j}\right)\right\} \\
& +\frac{1}{n^{2}-1}\left[\delta_{j}^{h}\left\{n\left(\bar{\varphi}_{r} \bar{R}_{i k}+\bar{a}_{r} \bar{K}_{i k}\right)+\left(\bar{\varphi}_{r} \bar{R}_{k i}+\bar{a}_{r} \bar{K}_{k i}\right)\right\}\right. \\
& \left.-\delta_{k}^{h}\left\{n\left(\bar{\varphi}_{r} \bar{R}_{i j}+\bar{a}_{r} \bar{K}_{i j}\right)+\left(\bar{\varphi}_{r} \bar{R}_{j i}+\bar{a}_{r} \bar{K}_{j i}\right)\right\}\right]
\end{aligned}
$$

By using the equation (4.1) in (4.4), we obtain

$$
\bar{\nabla}_{r} \bar{W}_{i j k}^{h}=\bar{\varphi}_{r} \bar{W}_{i j k}^{h}+\bar{a}_{r} \bar{N}_{i j k}^{h}
$$

where

$N_{i j k}^{h}=\bar{K}_{i j k}^{h}+\frac{\delta_{i}^{h}}{n+1}\left(\bar{K}_{j k}-\bar{K}_{k j}\right)+\frac{1}{n^{2}-1}\left[\delta_{j}^{h}\left(n \bar{K}_{i k}+\bar{K}_{k i}\right)-\delta_{k}^{h}\left(n \bar{K}_{i j}+\bar{K}_{j i}\right)\right]$.

Definition 15 If the projective curvature tensor $\bar{W}_{i j k}^{h}$ of $\bar{\nabla}$ satisfies the condition

$$
\bar{\nabla}_{r} \bar{W}_{i j k}^{h}=\bar{\gamma}_{r} \bar{W}_{i j k}^{h}
$$

where $\bar{\gamma}_{r}$ is the projectively recurrency vector, the Weyl manifold is called projectively recurrent WS manifold.

Definition 16 The Weyl manifold is called generalized projectively recurrent $W$ manifold, if the projective curvature tensor $W_{i j k}^{h}$ of $\nabla$ satisfies the condition

$$
\nabla_{r} W_{i j k}^{h}=\phi_{r} W_{i j k}^{h}+d_{r} N_{i j k}^{h}
$$

where $N_{i j k}^{h}$ is the generalized projectively recurrency tensor while $\phi_{r}$ and $d_{r}$ are the generalized projectively recurrency vectors of $W$ manifold. 
Theorem 17 A projectively recurrent WS manifold with the tensors $S_{i j}$ and $K_{i j}$ satisfying the conditions $\bar{\nabla}_{r} S_{i j}=\bar{\gamma}_{r} S_{i j}, \bar{\nabla}_{r} K_{i j}=\bar{\gamma}_{r} K_{i j}$, where $\bar{\gamma}_{r}$ is the projectively recurrency vector of WS manifold, is a generalized projectively recurrent $W$ manifold.

Proof. By differentiating covariantly (4.2) with respect to $\bar{\nabla}$, after some algeabric calculations, we get

$$
\begin{aligned}
\bar{\nabla}_{r} \bar{W}_{i j k}^{h} & =\bar{\nabla}_{r} W_{i j k}^{h}+\frac{2 \delta_{i}^{h}}{n+1} \bar{\nabla}_{r} S_{[k j]} \\
& +\frac{1}{n^{2}-1}\left[\delta_{k}^{h} \bar{\nabla}_{r} K_{i j}-\delta_{j}^{h} \bar{\nabla}_{r} K_{i k}\right]+g_{i j} g^{h l}\left(\bar{\nabla}_{r} S_{l k}\right) \\
& -g_{i k} g^{h l}\left(\bar{\nabla}_{r} S_{l j}\right) .
\end{aligned}
$$

In (4.7), $\bar{\nabla}_{r} W_{i j k}^{h}$ denotes the covariant derivative of the projective curvature tensor $W_{i j k}^{h}$ of W manifold with respect to $\bar{\nabla}$ given by

$$
\bar{\nabla}_{r} W_{i j k}^{h}=\nabla_{r} W_{i j k}^{h}+W_{i j k r}^{h}
$$

where

$$
\begin{aligned}
W_{i j k r}^{h} & =S^{m}\left(W_{m j k}^{h} g_{i r}+W_{i m k}^{h} g_{j r}+W_{i j m}^{h} g_{k r}\right)-\left(W_{r j k}^{h} S_{i}+W_{i r k}^{h} S_{j}+W_{i j r}^{h} S_{k}\right) \\
& +W_{i j k}^{m}\left(\delta_{r}^{h} S_{m}-g_{m r} S^{h}\right) .
\end{aligned}
$$

By substituting (4.8) into (4.7), we obtain

$$
\begin{gathered}
\bar{\nabla}_{r} \bar{W}_{i j k}^{h}-\frac{2 \delta_{i}^{h}}{n+1} \bar{\nabla}_{r} S_{[k j]}-\frac{1}{n^{2}-1}\left[\delta_{k}^{h} \bar{\nabla}_{r} K_{i j}-\delta_{j}^{h} \bar{\nabla}_{r} K_{i k}\right] \\
-g_{i j} g^{h l}\left(\bar{\nabla}_{r} S_{l k}\right)+g_{i k} g^{h l}\left(\bar{\nabla}_{r} S_{l j}\right)=\nabla_{r} W_{i j k}^{h}+W_{i j k r}^{h} .
\end{gathered}
$$

By the conditions $\bar{\nabla}_{r} S_{i j}=\bar{\gamma}_{r} S_{i j}, \bar{\nabla}_{r} K_{i j}=\bar{\gamma}_{r} K_{i j}$ and the Definition 15, (4.9) reduces to the equation

$$
\nabla_{r} W_{i j k}^{h}=\bar{\gamma}_{r} W_{i j k}^{h}-W_{i j k r}^{h}
$$

meaning that the manifold is a generalized projectively recurrent $\mathrm{W}$ manifold.

\section{References}

[1] Hayden, H.A., Subspaces of a space with torsion, Proc. London Math. Soc., $1932,34,27-50$.

[2] Yano, K., On semi symmetric metric connection, Rev. Roumaine Math. Pure Appl., 1970, 15, 1579-1586. 
[3] Imai, T., Hypersurfaces of a Riemannian manifold with semi symmetric metric connection, Tensor N.S., 1972, 23, 300-306.

[4] Imai,T., Notes on semi symmetric metric connections, Tensor N.S., 1972, 24, 293-296.

[5] Unal, F., Uysal, A., Weyl Manifolds with semi symmetric connections, Mathematical and Computational Applications, 2005, Vol.10, No:3.

[6] Enghiş, P., Relations between the tensors of various curvatures of a Dconnection, Proc. Of Symposium in Geometry (Cluj-Napoca and TirguMures), 1992, 99-102.

[7] Enghiş, P., Boer, M., Generalized recurrency in spaces with affine connection, Studia Univ. Babeş-Bolyai, Math., 1988, 33, 74-79.

[8] Norden, A., Affinely connected spaces, GRMFL, Moscow (in Russian), 1976. 